\title{
Hereditary Factor V Deficiency
}

National Cancer Institute

\section{Source}

National Cancer Institute. Hereditary Factor V Deficiency. NCI Thesaurus. Code C98938.

A very rare autosomal recessive inherited blood coagulation disorder characterized by deficiency of factor $V$, resulting in bleeding. 